\title{
THE BASIC PHYSICS OF HOT-STAR WINDS
}

\author{
STANLEY P. OWOCKI \\ Bartol Research Institute, University of Delaware, Newark, DE 19716 USA
}

\begin{abstract}
Hot-star winds are believed to be driven by line-scattering of the star's continuum radiation. This review summarizes the physics of this line-driving process and the resulting basic wind properties. I also briefly discuss the generation of wind structure, both at small scale due to inherent instabilities in the line-driving, and at large scales due to various rotational effects.
\end{abstract}

\section{Introduction}

Stellar winds from hot stars (i.e., spectral types 0 , B, and WR) have been known and studied for more than 20 years now. During this time, there has developed an extensive literature, including numerous reviews of both theory (e.g. Cassinelli 1979; Lucy 1985; Abbott 1988; Owocki 1990; Drew 1994) and observations (Conti 1978; Cassinelli \& Lamers 1987). (See also the monograph edited by Conti \& Underhill 1988.) Given this already rich collection of reviews, I will not attempt here to give any kind of exhaustive list of the literature. Instead, this review will provide a tutorial on the fundamental driving mechanism-line-scattering in the wind of the star's continuum radiation field-and what it implies for basic wind properties (e.g., mass-loss rate, $\dot{M}$, and terminal flow speed, $v_{\infty}$ ). I will also briefly summarize processes that can lead to wind structure and variability.

\section{The Line Force}

Hot-star winds are thought to be driven by line-scattering of the star's continuum radiation flux (Lucy \& Solomon 1970). Most modern models are based on the formalism developed by Castor, Abbott, \& Klein (1975; hereafter CAK) for including the ensemble effect of many lines. For simplicity let us assume (as did CAK) a point source of radially streaming radiation, but for now consider only a single, isolated line. The radiative acceleration associated with absorption of radiation in this line is then

$$
g_{\mathrm{rad}}(r)=g_{\mathrm{abs}}(r)=g_{\mathrm{thin}} \int_{-\infty}^{\infty} d x \phi\left(x-v(r) / v_{\mathrm{th}}\right) e^{-t(x, r)}
$$

where $v$ and $v_{\text {th }}$ are the flow speed and ion thermal speed, $\phi$ is the lineprofile function, and $x \equiv\left(\nu-\nu_{\mathrm{L}}\right) / \Delta \nu_{\mathrm{D}}$ is the frequency displacement from line center, measured in Doppler-units in the star's rest frame. The frequency integration corrects the optically thin acceleration $g_{\text {thin }} \equiv \kappa_{\mathrm{L}} L_{\nu} \Delta \nu_{\mathrm{D}} / 4 \pi c r^{2}$ for 
self-absorption, with the optical depth $t(x, r)$ at radius $r$ given by

$$
t(x, r)=\int_{R_{*}}^{r} \kappa_{\mathrm{L}} \rho\left(r^{\prime}\right) \phi\left(x-v\left(r^{\prime}\right) / v_{\mathrm{th}}\right) d r^{\prime} .
$$

Here $\kappa_{\mathrm{L}}$ is the line mass-absorption coefficient, and $L_{\nu}$ is the stellar luminosity at the line frequency $\nu$.

For a scattering line, there is, in principle, an additional force component associated with the diffuse, scattered radiation field; however, in a smooth, supersonic flow such scattering is nearly fore-aft symmetric (Sobolev 1960), which implies that the diffuse force is quite small, of order $v_{\text {th }} / v$ times $g_{\text {abs }}$ (Castor 1974). Another implication of this so-called 'Sobolev approximation' for supersonic flow is that the variation in the integrand of Eq. (2) is dominated by the Doppler-shift associated with changes in the velocity $v\left(r^{\prime}\right)$; by switching the variable of integration to the comoving frame frequency $x^{\prime} \equiv x-v\left(r^{\prime}\right) / v_{\text {th }}$, we thus find

$$
t(x, r) \approx \tau \int_{x-v(r) / v_{t h}}^{\infty} \phi\left(x^{\prime}\right) d x^{\prime}
$$

where the Sobolev optical depth $\tau \equiv \kappa_{\mathrm{L}} \rho v_{\text {th }} /(d v / d r)$ represents a collection of spatial variables that are assumed to be approximately constant over a Sobolev length $L \equiv v_{\text {th }} /(d v / d r)$. For example, in the case of a smooth, steady flow this means that the density scale length

$$
H \equiv \frac{\rho}{d \rho / d r} \approx \frac{v}{d v / d r} \gg \frac{v_{\text {th }}}{d v / d r} \equiv L,
$$

and hence that $v \gg v_{\text {th }}$. Since the ion thermal speed is on the order of the sound speed $a$, we can expect that the approximation will be well satisfied in the supersonic portions of a smooth flow. Applying Eq. (3) to Eq. (1) we see that both required frequency integrations can now be done analytically, yielding

$$
g_{\mathrm{rad}}(r)=g_{\mathrm{thin}} \frac{1-e^{-\tau}}{\tau}
$$

Note that for a weak line $\tau \ll 1$ we recover the optically thin expression, while for a strong line $\tau \gg 1$ we obtain the optically thick form

$$
g_{\mathrm{rad}}(r)=\frac{g_{\mathrm{thin}}}{\tau}=\frac{\nu_{\mathrm{L}} L_{\nu}}{L_{*}} \frac{L_{*}}{4 \pi \rho r^{2} c^{2}} \frac{d v}{d r} .
$$

The latter is independent of the opacity $\kappa_{L}$, depending instead on the velocity gradient $d v / d r$. Once a line is optically thick, all photons shifted into resonance with it are scattered, and further increases in the opacity only push the first scattering further into the blue wing. The number of photons 
scattered per unit length is thus independent of the opacity, and depends instead on the slope of the photon red-shift, which, in turn, depends on the velocity gradient. Normally, such a velocity gradient is part of an inertial term that represents the acceleration that results from a driving force, but here we see that it helps determine the driving force. In general terms, just as the optically thin line force helps reduce the effect of gravity on the fluid, so does the optically thick line force reduce the effect of inertia.

A simple consideration of just the inertial force balance enables us to estimate the mass-loss rate that results from such line-driving. The acceleration is just

$$
v \frac{d v}{d r}=g_{\mathrm{rad}}=\frac{\nu_{\mathrm{L}} L_{\nu}}{L_{*}} \frac{L_{*}}{4 \pi \rho r^{2} c^{2}} \frac{d v}{d r}
$$

which implies that the mass-loss rate $\dot{M}_{1}$ associated with driving by a single, isolated line is

$$
\dot{M}_{1}=4 \pi \rho v r^{2}=\frac{\nu_{\mathrm{L}} L_{\nu}}{L_{*}} \frac{L_{*}}{c^{2}} .
$$

Since $\nu_{\mathrm{L}} L_{\nu} / L_{*} \approx 1$, we see that the wind mass-loss rate driven by a single line is only about the photon mass-loss rate! It is essentially for this reason that the original Lucy \& Solomon (1970) model, which considered the effect of driving in only a few strong lines, yielded such low mass-loss rates.

\section{The CAK Model: Driving by a Line Ensemble}

A major advance of the CAK model was to develop a formalism for efficiently including the cumulative effect of a large ensemble of lines, which they effectively assumed to have a flux-weighted number distribution that was a power law in opacity, i.e., $N(\kappa) \sim \kappa^{\alpha-2}$, where $0<\alpha<1$. Assuming the lines do not significantly overlap, the cumulative force can then be computed by integrating the expression (1) over this number distribution. Applying, as before, the Sobolev approximation, one obtains the CAK line-force

$$
g_{\mathrm{CAK}}=\frac{K L_{*}}{r^{2}}\left(\frac{1}{\rho} \frac{d v}{d r}\right)^{\alpha}
$$

where $K$ is a normalization constant for the line-distribution (related to the CAK constant $k$ by $K=k\left(\kappa_{\mathrm{e}}^{1-\alpha} / 4 \pi c v_{\text {th }}{ }^{\alpha}\right)$, where $\kappa_{\mathrm{e}}$ is the electron scattering opacity.)

Gas pressure forces play a relatively minor role in driving these winds, and so we can derive the basic wind characteristics by considering just the balance among inertial, gravitational, and radiative forces, ignoring gas pressure altogether. The requirement of momentum balance thus reduces to the condition,

$$
F \equiv w^{\prime}+1-C w^{\prime \alpha}=0,
$$


where $w^{\prime} \equiv r^{2} v v^{\prime} / G M_{*}$ and

$$
C \equiv \frac{K}{G M_{*}}\left(\frac{4 \pi G M_{*}}{\dot{M} c}\right)^{\alpha} .
$$

Depending on the value of $\dot{M}$, the constant $C$ will be such that Eq. (10) will admit either 0,1 , or 2 solutions. The critical case with one solution has the property that $F=\partial F / \partial w^{\prime}=0$, which implies the critical values $w_{\mathrm{c}}^{\prime}=\alpha /(1-\alpha)$ and $C_{\mathrm{c}}=(1-\alpha)^{\alpha-1} / \alpha^{\alpha}$. Since Eq. (10) has no explicit spatial dependence, this critical value $w_{c}^{\prime}$ must hold throughout the flow. Integrating $w_{c}^{\prime}$ from the stellar radius $R_{*}$, we thereby obtain the usual "CAK velocity law",

$$
v_{\mathrm{CAK}}(r)=v_{\infty} \sqrt{1-\frac{R_{*}}{r}},
$$

where the asymptotic wind speed is given by

$$
v_{\infty} \equiv \sqrt{\frac{2 \alpha \mathrm{G} M_{*}}{(1-\alpha) R_{*}}}=\sqrt{\frac{\alpha}{1-\alpha}} v_{\text {esc }} .
$$

Likewise, if we solve the definition (11) for the mass-loss rate and apply the critical value $C_{\mathrm{c}}$, we obtain the CAK mass-loss rate formula,

$$
\dot{M}_{\mathrm{CAK}}=4 \pi \alpha\left(\frac{G M_{*}}{1-\alpha}\right)^{1-1 / \alpha}\left(K L_{*}\right)^{1 / \alpha}
$$

These are just the relations derived by CAK for the case of a point-star; taking account of the finite angular size of the stellar disk tends to make the mass-loss rate lower, the velocity law shallower, and the terminal speed higher (Friend \& Abbott 1986; Pauldrach, Puls, \& Kudritzki 1986). The effect of retaining a finite gas pressure is comparatively small, as is the effect of using a full comoving frame transfer solution instead of the CAK/Sobolev line force (9) (Pauldrach et al. 1986). Recent further efforts (e.g. Gabler et al. 1989) have focussed on developing unified wind-atmosphere models that abandon the artificial distinction between "core" and "halo", and that solve for the basic wind parameters $k$ and $\alpha$ from a more complete treatment of the NLTE statistical equilibrium (Pauldrach 1987). A major overall goal of these efforts is to carry out "quantitative spectroscopy", i.e., to compare quantitatively theoretical and observed spectra in order to infer more precisely and reliably the basic parameters of both wind and star. Further details of these efforts are given in the review by Kudritzki \& Hummer (1990) and references therein. 


\section{Multi-Line Scattering in Overlapping Lines}

For a typical OB supergiant model (e.g., $\zeta$ Pup), the CAK mass-loss rate is $\sim 100$ times the single line value, implying that there are effectively about 100 optically thick lines spread throughout the spectrum. On the other hand, note from Eq. (13) that the terminal flow speed $v_{\infty}$ scales with surface escape speed $v_{\text {eac }}$, and is independent of the number of strong lines. Typically $v_{\infty}$ is on the order of $1 \%$ of the speed of light, so that, quite coincidentally, in the CAK model

$$
\dot{M} v_{\infty} \approx \frac{L_{*}}{c}\left(N_{\text {thick }} \frac{v_{\infty}}{c}\right) \approx \frac{L_{*}}{c}
$$

which is often referred to as the "single scattering limit". This means that, fortuitously, the CAK model is just barely self-consistent in ignoring line overlap, if one assumes the lines are smoothly spread throughout the stellar spectrum.

In reality, of course, there is always some line overlap, arising both from chance frequency coincidences of lines as well as from the tendency for lines to occur in multiplets. An early but quite elegant examination of the dynamical effects of such overlap was carried out by Friend \& Castor (1983), who derived an extension of the CAK model to include overlap under the assumption that the line frequency spacing is Poisson distributed. Although this ignored the tendency of lines to occur in multiplets, it demonstrated quite clearly the major effect that overlapping lines can drive mass-loss in excess of the single scattering limit, i.e., with $\dot{M} v_{\infty}>L_{*} / c$. Similar results were obtained for more realistic line lists by Abbott \& Lucy (1985) from a MonteCarlo calculation, and by Puls (1987), who obtained approximate solutions of the coupled system of radiative transfer equations.

For $\mathbf{O}$ and $\mathrm{B}$ stars such overlap effects are typically minor, but they probably play a much more fundamental role in the Wolf-Rayet stars, which are inferred observationally to have $\dot{M} v_{\infty} /\left(L_{*} / c\right) \approx 5--50$ (Abbott \& Conti 1987). Lucy \& Abbott (1993) have recently applied their Monte-Carlo code to derive mass-loss rates for a multi-line scattering wind model assuming a fixed wind velocity law. For typical WR star parameters they were able to achieve $\dot{M} v_{\infty} \approx 10 L_{*} / c$. These results make it clear that there is nothing fundamental about the single scattering limit, and that this limit can be exceeded by a large factor, if photons can be sufficiently trapped in the envelope. Thus, although the difficulty in explaining the large inferred massloss rates for Wolf-Rayet stars is often characterized as the "momentum" problem, it really is more of an "opacity" problem. In the Lucy \& Abbott (1993) model, there is a tendency for the ionization balance to adjust so that there is always lots of line opacity near the peak of the photon spectrum, even as this spectrum shifts to longer wavelengths at larger radial distances in the cooling envelope. A further physical discussion of how such photon 
trapping enhances momentum deposition in the wind is given by Gayley \& Owocki (1994).

\section{Generation of Wind Structure}

There is much evidence that hot-star winds are not the smooth, steady, spherically symmetric outflows envisaged in the above models, but rather are highly variable and structured on both relatively large and small scales. Such structure could arise in several ways, including perturbations from stellar pulsation, rotational modulation associated with underlying atmospheric structure (possibly magnetic), or simply from the intrinsic instabilities in the wind itself.

The most direct evidence for relatively large-scale structure comes from the variable "discrete absorption components"(DAC) of unsaturated UV lines, which are observed in IUE monitoring campaigns to form at $v \sim v_{\infty} / 3$ and then to migrate to higher frequencies. Such DAC's typically recur quasiregularly, with both a recurrence and evolution time scaling roughly with the inverse of the projected rotation velocity, typically a day or two (Prinja 1988; Kaper 1993; see also contributions by Henrichs and by Prinja in these Proceedings). They are thought to arise from accelerating wind density enhancements that are large enough (i.e., a stellar radius or so) to cover a substantial fraction of the stellar disk. Mullan $(1984 ; 1986)$ has proposed that these could arise from "co-rotating interaction regions" (CIR's) between fast and slow speed wind streams. In the solar wind, such wind speed differences can arise from the differences in flow divergencies associated with magnetic field structure in the lower solar corona. MacGregor (1988) has shown that changes in flow divergence can have a similarly large influence on the wind speeds in a line-driven flow. For normal OB stars, observational upper limits on the global magnetic field are typically about $B<100 G$, but even fields below this could have an important effect. In general a field can divert a flow up to the Alfven critical point, which for these winds occurs at a velocity $v \approx 1500 \mathrm{~km} \mathrm{~s}^{-1}\left(B_{100}^{2} r_{12}^{2} / \dot{M}_{-6}\right)$, where $B_{100} \equiv B / 100 \mathrm{G}, r_{12} \equiv r / 10^{12} \mathrm{~cm}$, and $\dot{M}_{-6} \equiv \dot{M} / 10^{-6} M_{\odot} \mathrm{y}^{-1}$. This implies that undetected fields, e.g. with $B \gtrsim 10$ $G$, could have quite a significant dynamical influence on the acceleration of OB winds.

The relatively low signal to noise $(\sim 20)$ in IUE spectra has severely limited our ability to directly detect wind fluctuations on a smaller scale in UV lines for OB stars. However, such small-scale fluctuations are directly measured in the much higher signal to noise $(\sim 500)$, ground-based observations of optical emission lines formed in WR star winds (McCandliss 1991; Robert 1991), and these have been interpreted as implying the existence of a "turbulent" spectrum of relatively small-scale structures in these winds (Moffat et al. 1988, 1993). Furthermore, even in OB winds, there is indi- 
rect evidence for such small-scale structure, in the form of the nearly black absorption troughs of saturated UV lines, which seem to require a multiply nonmonotonic velocity field (Lucy 1982).

It seems likely that such small-scale, "stochastic" structure could arise naturally from the strong instability of the line-driving mechanism itself (Rybicki 1987). A small-scale increase in radial flow speed Doppler-shifts the local line-frequency out of the absorption shadow of intervening material, leading to an increased line-force which then tends to further increase the flow speed. Formal stability analyses (MacGregor, Hartmann, \& Raymond 1979; Carlberg 1980; Owocki \& Rybicki 1984, 1985) yield a growth rate nearly a hundred times the wind expansion rate, implying that even very small amplitude perturbations should quickly become nonlinear.

Since the instability occurs primarily for perturbations at a scale near and below the Sobolev length $L$ (cf. Eq. (4)), it cannot be analyzed in terms of the simple, local force expressions (5) or (9), which are based on the Sobolev approximation that all variations occur on a scale much larger than $L$. Rather it requires expensive computation of numerical integrals occurring in nonlocal force expressions, either based on a pure-absorption approximation (Owocki, Castor, \& Rybicki 1988; hereafter OCR), or the "Smooth Source Function" approximation (Owocki 1991) for line-scattering. Numerical simulations of the nonlinear evolution (OCR; Puls, Owocki, \& Fullerton 1994; Feldmeier 1993, 1994) show a wind embedded with multiple reverse shocks and dense shells. Recent models with greater resolution to resolve relatively high-frequency structure have synthetic spectra that reproduce quite well the broad, black absorption troughs observed in saturated lines (Owocki, Puls, \& Fullerton 1994; Puls et al. 1994). The resulting wind shocks also seem to provide a potential explanation for the soft X-ray emission typically observed from OB stars (Cooper \& Owocki 1991, 1993). Work is also underway to try to generalize such simulations to WR winds, with the aim to model the small-scale fluctuations seen in optical wind lines (Gayley \& Owocki 1994).

In unsaturated UV lines, the dense shells that form in these instability simulations also give rise to multiple narrow absorption features which superficially resemble DAC's, but which have acceleration time scales that are more characteristic of the mean wind (i.e., with velocity law exponent $\beta \approx 1$ ) than of the much more slowly evolving DAC's (for which typically $\beta \approx 3--10$ ). Considering also that their recurrence is nearly stochastic, it seems unlikely that such intrinsic, small-scale wind instabilities could be the direct cause of the semi-regularly recurring DAC's. Emphasis has thus shifted to trying to simulate such DAC's as resulting from a relatively large scale disturbance at the wind base. For example, a recent simulation (Owocki, Puls, \& Fullerton 1994) shows that, if the density in the lower wind (at $v \approx 100 \mathrm{~km} \mathrm{~s}^{-1}$ ) is artificially increased by just a factor of two, then the 
outer wind develops a large, slow-moving dense shell with roughly the same slow acceleration time scale seen in DAC's.

Such time-dependent instability models have so far been restricted to 1-D, owing to the great computational expense of computing the nonlocal radiative force. In a more realistic 3-D model, the relatively narrow shells that form in such 1-D models would almost certainly be broken into small-scale clumps which, due to global averaging, would have much weaker spectral line signatures. However, even though the 3-D wind structure would thus not have the lateral phase coherence of a strictly 1-D model, it still seems likely that velocity fluctuations would be predominately $1-D$ radially polarized, since 3-D linear stability analyses (Rybicki, Owocki, \& Castor 1990) indicate that lateral velocity fluctuations are strongly damped by the linedrag effect of the diffuse radiation field (Lucy 1984). There appears to be some evidence for such a predominance of radial velocity fluctuations in WR winds (McCandliss 1991; Robert 1991).

If one is interested in only relatively large-scale wind structure, i.e., larger than a Sobolev length $L$, then it is possible to carry $2-\mathrm{D}$ or 3 -D simulations using the simple, local CAK/Sobolev force expression (9), which is much cheaper to compute than the nonlocal expressions needed to study smallscale instabilities. Owocki, Cranmer, \& Blondin (1994) have recently carried out 2-D hydrodynamical simulations of CAK winds from rapidly rotating stars, in order to test the Bjorkman \& Cassinelli (1993) "Wind Compressed Disk" (WCD) model. With some modifications, these simulations basically confirm the original WCD idea, but do show some interesting new features, e.g., inner disk infall. Such WCD models have a natural appeal for explaining the $\mathrm{H} \alpha$ emission from the relatively rapid rotating Be stars, which have long been associated with disks. Unfortunately, as discussed in the paper by Bjorkman in these Proceedings, it now appears that, the simple wind compression effect results in only a relatively low-density, optically thin disk that seems inadequate to explain either the observed $\mathrm{H} \alpha$ emission or the observed optical continuum polarization from such stars. To overcome these limitations, it may be worthwhile for future models to consider the possible role of magnetic fields in further guiding the wind toward the equator and in spinning up the disk.

\section{Acknowledgements}

This work was supported in part by NSF grant AST 91-15136 and NASA grants NAGW-2624 and NAG5-1567, and by an allocation of supercomputer time from the San Diego Supercomputer Center. I thank A. Fullerton and S. Cranmer for helpful comments. 


\section{References}

Abbott, D.C.: 1988, in Pizzo, V.J., Holzer, T.E., Sime, D.G., eds., Proceedings of the Sixth International Solar Wind Conference, Vol. 1, NCAR: Boulder, 149.

Abbott, D.C. and Conti, P.S.: 1987, Ann. Rev. Astron. Astrophys. 25, 113.

Abbott, D.C. and Lucy, L.B.: 1985, Astrophys. J. 288, 679.

Bjorkman, J.E. and Cassinelli, J.P.: 1993, Astrophys. J. 409, 429.

Carlberg, R.G.: 1980, Astrophys. J. 241, 1131.

Cassinelli, J.P.: 1979, Ann. Rev. Astron. Astrophys. 17, 275.

Cassinelli, J.P. and Lamers, H.G.J.L.M.: 1987, in Kondo, Y. et al. eds., Exploring the Universe with the IUE Satellite, Reidel: Dordrecht, 140.

Castor, J.I.: 1974, Mon. Not. Roy. Astr. Soc. 169, 279.

Castor, J.I., Abbott, D.C. and Klein, R.I.: 1975, Astrophys. J. 195, 157 (CAK).

Conti, P.S.: 1978, Ann. Rev. Astron. Astrophys. 16, 371.

Conti, P.S. and Underhill, A.B.: 1988, O Stars and Wolf-Rayet Stars, NASA: Washingtoa.

Cooper, R.G. and Owocki, S.P.: 1991, in Drissen, L., Leitherer, C. and Nota, A., eds., Nonisotropic and Variable Outflows from Stars, Astron. Soc. Pacific: San Francisco, 281.

Cooper, R.G. and Owocki, S.P.: 1993, in Moffat, A. et al. eds., Instability and Variability in Hot Star Winds, Kluwer: Dordrecht, in press.

Drew, J.E.: 1994, in Clegg, R., Meikle, P. and Stevens, I., eds., Circumstellar Media in the Late Stages of Stellar Evolution, Cambridge Univ. Press: Cambridge, in press.

Feldmeier, A.: 1993, Ph. D. Thesis, Univ. Munich: Germany.

Feldmeier, A.: 1994, in Moffat, A. et al. eds., Instability and Variability in Hot Star Winds, Kluwer: Dordrecht, in press.

Friend, D.B. and Abbott, D.C.: 1986, Astrophys. J. 311, 701.

Friend, D.B. and Castor, J.I.: 1983, Astrophys. J. 272, 259.

Gabler, R., Gabler, A., Kudritzki, R.P., Puls, J. and Pauldrach, A.W.A.: 1989, Astron. Astrophys. 226, 162.

Gayley, K. and Owocki, S.P.: 1994, Astrophys. J. , submitted.

Kaper, L.: 1993, Ph.D. Thesis, Univ. Amsterdam: The Netherlands.

Kudritzki, R.P. and Hummer, D.G.: 1990, Ann. Rev. Astron. Astrophys. 28, 303.

Lucy, L.B.: 1982, Astrophys. J. 255, 286.

Lucy, L.B.: 1984, Astrophys. J. 284, 351.

Lucy, L.B.: 1985, in Mihalas, D. and Winkler, K-H.A., eds., Radiation Hydrodynamics in Stars and Compact Objects, IAU Colloq. No. 89, Springer: Berlin, 75.

Lucy, L.B. and Abbott, D.C.: 1993, Astrophys. J. 405, 738.

Lucy, L.B. and Solomon, P.M.: 1970, Astrophys. J. 159, 879.

MacGregor, K.B.: 1988, Astrophys. J. 327, 794.

MacGregor, K.B., Hartmann, L. and Raymond, J.C.: 1979, Astrophys. J. 231, 514.

McCandliss, S.R.: 1991, in Drissen, L., Leitherer, C. and Nota, A., eds., Nonisotropic and Variable Outflows from Stars, Astron. Soc. Pacific: San Francisco, 214.

Moffat, A.F.J., Drissen, L., Lamontagne, R. and Robert, C.: 1988, Astrophys. J. 334, 1038.

Moffat, A.F.J., Lepine, S., Henriksen, R.N. and Robert, C.: 1993, Kinematics and Dynamics of Diffuse Astrophysical Media, Astophys. Space Sci., in press.

Mullan, D.J.: 1984, Astrophys. J. 283, 303.

Mullan, D.J.: 1986, Astron. Astrophys. 165, 157.

Owocki, S.P.: 1990, Rev. Modern Astron. 3, Springer: Berlin, 98.

Owocki, S.P.: 1991, in Crivellari, L., Hubeny, I. and Hummer, D.G., eds., Stellar Atmospheres: Beyond Classical Models, Kluwer: Dordrecht, 235.

Owocki, S.P. and Rybicki, G.B.: 1984, Astrophys. J. 284, 337.

Owocki, S.P. and Rybicki, G.B.: 1985, Astrophys. J. 299, 265.

Owocki, S.P., Castor, J.I. and Rybicki, G.B.: 1988, Astrophys. J. 335, 914 (OCR).

Owocki, S.P., Cranmer, S.R. and Blondin, J.B.: 1994, Astrophys. J. , in press.

Owocki, S.P., Puls, J. and Fullerton, A.W.: 1994, in Moffat, A. et al. eds., Instability and 
Variability in Hot Star Winds, Kluwer: Dordrecht, in press.

Pauldrach, A.: 1987, Astron. Astrophys. 183, 285.

Pauldrach, A. and Puls, J.: 1990, Astron. Astrophys. 237, 409.

Pauldrach, A., Puls, J. and Kudritzki, R.P.: 1986, Astron. Astrophys. 164, 86.

Prinja, R.K.: 1988, Mon. Not. Roy. Astr. Soc. 231, 21P.

Puls, J.: 1987, Astron. Astrophys. 184, 227.

Puls, J., Owocki, S.P. and Fullerton, A.W.: 1994, Astron. Astrophys. , in press.

Puls, J., Feldmeier, A., Springmann, U., Owocki, S.P. and Fullerton, A.W.: 1994, in Moffat,

A. et al. eds., Instability and Variability in Hot Star Winds, Kluwer: Dordrecht, in press.

Robert, C.: 1991, Ph.D. Thesis, Univ. de Montreal: Canada.

Rybicki, G.B.: 1987, in Lamers, H.J.G.L.M. and de Loore, C.W.H., eds., Instabilities in Luminous Early Type Stars, Reidel: Dordrecht, 175.

Rybicki, G.B., Owocki, S.P. and Castor, J.I.: 1990, Astrophys. J. 349, 274.

Sobolev, V.V.: 1960, Moving Envelopes of Stars, Harvard Univ. Press: Cambridge.

\section{Discussion}

Lafon: (1) The problem of comparing $L / c$ and $\dot{M} v_{\infty}$ is not well posed! Indeed momentum is a vectorial quantity and should be added as a vector so that the net flux of momentum from the star is always zero in azimuthal and plane (equatorial symmetry), or in spherical symmetry. Thus there is no problem with finding ratios $M v_{\infty} c / L$ of the order 50 or larger.

(2) The "mass-loss rate", $\dot{M}$, is only defined in steady state. Otherwise some amount of matter elevated from the surface of the star can fall back to the star later. (This is a well known phenomenon for shock perturbed atmospheres of evolved stars.) One should not talk of "mass-loss variability" where observing line variation for lines formed or changed in the wind.

(3) Instabilities, even in spherically or azimuthally symmetric models, should be investigated in three dimensions, even if the global structure of the wind is not perturbed on large scales. First the instabilities may produce blobs or clumps instead of shells. Then one should be cautious with comparisons with the solar wind in which large scale structures are maintained by freezing of magnetic field, contrary to what occurs in hot star winds.

Owocki: (1) As noted in my talk, I basically agree that, in principle, $\dot{M} v_{\infty} c / L$ can become very large. It requires, however, a large opacity distributed throughout the spectrum to keep photons trapped in the wind, and, in practice, this can be quite difficult to achieve. This is why I prefer to characterize WR wind models as suffering an "opacity" rather than a "momentum" problem.

(2) If one simply chooses to define $\dot{M} \equiv 4 \pi \rho v r^{2}$ at each location in the wind, then this quantity can vary in time or space in a time-dependent, or a non-spherically symmetric wind model. Such variations in local mass flux often have quite distinct, detectable consequences in observed spectra, and so I believe it is quite appropriate to speak in this way of "mass-loss variability". 
(3) Rybicki, Owocki \& Castor (1990) investigated the linear instability of winds in 3-D, but so far nonlinear simulations have had to be limited to 1-D, simply because of the great computational expense of computing the nonlocal line force. As for the role of magnetic fields, this in my opinion is still an open question, since large scale fields with $B<100$ Gauss, roughly the observational upper limit for most $O B$ winds, could have dynamical effects as strong or stronger as those seen in the solar wind.

Harmanec: You mentioned that the $\alpha$ coefficient of proportionality is basically given by the specific mixture of absorbing lines. Now, many Be stars exhibit long-term spectral variations, developing strong shell lines at certain epochs. Would you agree that the $\alpha$ vary correspondingly and that this could be a way to further test the theory?

Owocki: The basic line-driving parameters $\alpha$ and $k$ can switch abruptly with stellar conditions, such as in the Pauldrach \& Puls (1990) "bi-stability" mechanism. Determining the role, if any, such switching may play in the epochal variations you mention requires further investigation.

Kaper: Which conditions have to fulfilled to get fast and slow moving streams in a radiatively driven wind?

Owocki: Within the context of a steady, CAK/Sobolev type line-driving, MacGregor (1988) showed that a faster-than-radial flow divergence-such as can occur in region of open, diverging magnetic field-can lead to more than a factor of two increase in asymptotic flow speed. Within the context of time-dependent models, inducing base variations in, e.g., mass flux, can lead to even larger variations in wind speed, owing to the nonlocal, nonlinear character of the radiative driving. My own expectation is that achieving flow speed variations is, in principle, much easier in hot-star winds than in the solar wind, where factor two fluctuations in speed are quite common.

Smith: Your wind-velocity diagram for a B2.5e star showed low wind velocities in the equatorial zone. Why, then, do we observe high velocity DAC's in spectra of Be-stars viewed nearly edge-on?

Owocki: The model I showed (see Fig. 2 of Owocki, Cranmer \& Blondin 1994 ) is just a simple simulation meant primarily to investigate the Bjorkman/Cassinelli Wind Compressed Disk effect, but it would still allow for relatively high-velocity DAC's arising in the flow just outside the very thin disk, which would still be seen projected against the star.

Prinja: Based on your latest calculations, do you predict that the intrinsic wind instabilities (resulting in small-scale structure) would be observable as time-dependent variations in the wind profiles of OB stars?

Owocki: The small-scale structure would necessarily produce direct variability that could only be detected at sufficiently high signal to noise, prob- 
ably more than 100 , and in any case much higher than typical for IUE. The presence of small scale structure is nonetheless implied by the overall profile shape, viz. the width and blackness of absorption troughs in saturated lines, which I do believe can only be produced by nonmonotonic velocity variations with a high spatial frequency.

Mourand: Have you plans to compute brightness distributions in your model in order to make direct comparisons with interferometric data?

Owocki: We do hope in general to develop 2-D and 3-D radiative transfer codes to synthesize from these models a wide range of observational diagnostics, including line-spectra, polarization, and, eventually, interferometry. 